\title{
Predictors of symptomatic remission in patients with first-episode schizophrenia: A 16 years follow-up study
}

\author{
Carlo Marchesi ${ }^{\mathrm{a}, \mathrm{b}, *}$, Andrea Affaticati ${ }^{\mathrm{b}}$, Alberto Monici ${ }^{\mathrm{b}}$, Chiara De Panfilis ${ }^{\mathrm{a}, \mathrm{b}}$, \\ Paolo Ossola ${ }^{a}$, Matteo Tonna ${ }^{\mathrm{b}}$ \\ ${ }^{a}$ Department of Neuroscience, Unit of Psychiatry, University of Parma, Italy \\ ${ }^{\mathrm{b}}$ Mental Health Department, Local Health Agency, Parma, Italy
}

\begin{abstract}
Objective: Recent views on schizophrenia outcome and treatment suggest that symptomatic remission is possible, and a definition of remission has been proposed by the Remission in Schizophrenia Working Group (RSWG).

This study evaluated whether patients who achieved remission after several years of illness $(\mathrm{R})$ showed psychopathological differences at the onset of their disorder compared to non-remitted (NR) patients.

Method: Forty-eight patients with first-episode schizophrenia were evaluated with the Positive and Negative Symptoms Scale (PANSS) both at the onset of illness and after a mean period of 16 years. Patients were defined as R or NR according to the RSWG criteria.

Results: Eighteen patients (37.5\%) were classified as R at follow-up. At onset, R patients showed a lower illness severity, less severe negative and general psychopathology symptoms compared to NR. Furthermore, they underwent fewer psychotic episodes than NR over the course of follow-up. Remission was predicted by lower severity of negative and general psychopathology symptoms at onset and by lesser number of psychotic episodes during follow-up.

Conclusions: The symptomatic remission may be a viable outcome in schizophrenia, particularly for patients with a mild illness and less severe negative symptoms at onset and with few psychotic episodes over time.
\end{abstract}

(C) 2014 Elsevier Inc. All rights reserved.

\section{Introduction}

Since the description of "dementia praecox" by Kraepelin [1], schizophrenia has been conceptualized as a chronic illness leading to mental deterioration, lack of volition and social incompetence with no hope for sustained remission or recovery [2]. Initially, diagnosis and prognosis were essentially the same [3] and any dramatic improvement or recovery during the course of the illness was viewed as signs of earlier misdiagnosis [4]. More recently, long-term follow-up studies have shown that the course of schizophrenia is highly heterogeneous $[5,6]$; outcome in schizophrenia is a complex and multideterminate phenomenon and the ability of this diagnosis

\footnotetext{
* Corresponding author at: Università di Parma, Dipartimento di Neuroscienze, Unità di Psichiatria, Ospedale-Padiglione Braga, Via Gramsci 14, 43125 Parma, Italy. Tel.: +390521 903594; fax: +390521347047.

E-mail address: carlo.marchesi@unipr.it (C. Marchesi).
}

to specifically predict a poor outcome has often been overstated [7]. For decades, the lack of generally accepted definition of treatment response, remission or recovery, which were considered largely impossible, represented a major problem for schizophrenia research [8]. Thus, the comparability of the research findings was limited by the variability of the criteria used in different studies [9]. In clinical practice the so-called "rule of thirds" became popular: in a group of schizophrenic patients one-third improves, one third deteriorates and one third has an intermediate course. But "the rule of thirds" did not have an empirical basis [10]. In order to improve the understanding of schizophrenia and its treatment options, the Remission in Schizophrenia Working Group (RSWG) [11] posited that "symptomatic remission is a definable concept and an achievable stage in the treatment of schizophrenia", since "psychosocial therapies and rehabilitation are most effective when positive and negative symptoms are adequately controlled". The RSWG proposed specific criteria for symptomatic remission with the 
aim to facilitate comparisons of effectiveness across the range of available therapeutic options and to support a positive, longer-term approach regarding outcome for patients with schizophrenia. Standardized RSWG consensus criteria for remission are based on severity of core psychopathological symptoms as assessed by the PANSS (symptomatic remission) which is sustained over a minimum of 6 months (time criterion) [11]. According to these criteria, a percentage of patients ranging from $45 \%$ to $70 \%$ were defined as remitters at some point during the course of their illness [12]. Concerning the identification of early predictors of symptomatic remission, a shorter duration of untreated psychosis (DUP), a better premorbid adjustment, lower illness severity at baseline, early symptomatic improvement, medication adherence and remitted substance abuse are thought to increase the likelihood for remission [12]. However, some of the existing studies failed to fully apply the RSWG criteria, for example by omitting the time criterion or by using different measures of symptom severity [12]. In addition, most of the available studies assessed the remission rate over a short time period, while only few studies employed a follow-up longer than 2 years. Thus, as claimed by Lambert [12], "comparability in terms of validity of criteria as well as frequencies and predictors of remission is limited", and further research is warranted in this area.

Patients achieving RSWG remission criteria are likely to be highly heterogeneous in terms of their psychopathological features. Specifically, they could show at the onset peculiar patterns and varying severity of the negative, disorganized and psychoticism (or reality distortion) dimensions, which represent related but distinguishable components of the schizophrenia process $[11,13,14]$. Previous studies reported that more severe negative symptoms at baseline robustly predict poor outcome [2,15-18]. However, the role of any specific psychopathological pattern in predicting long-term remission is still far to be established: while one study [19] confirmed that higher PANSS negative symptom scores at baseline predicted a lower likelihood of remission at 6-year follow-up, another study [20] did not report any psychopathological differences at the early stage of illness between remitted and non-remitted patients at a 7-year follow-up. Such conflicting findings may be partly due to the different phases of illness in which patients were evaluated, i.e., firstepisode [20] versus chronic schizophrenia [19]. Assessing symptom severity at different time points during the course of the illness may be critical for the evaluation of the course and stability of psychopathological dimensions.

Therefore, the present study aimed to investigate whether the severity of positive, disorganized and negative symptoms assessed at onset in first-episode patients with schizophrenia predicted remission (RSWG criteria) after several years (16 on average) of illness. In addition, a secondary aim was to evaluate how these three psychopathological dimensions changed over time.

\section{Materials and method}

\subsection{Sample}

The study participants were recruited from patients who were consecutively admitted to the Psychiatric Clinic of the University of Parma, from January 1995 to December 1999 for a first psychotic episode. This study is a part of a more extensive evaluation of long-term outcome in patients with a first psychotic episode approved by the Local Ethic Committee.

Patients were included in the study if: 1) they were aged over 17 years; 2) they were hospitalized for the first time in a psychiatric unit for a first psychotic episode; 3) they were discharged with a diagnosis of schizophrenia, according to the DSM-IV; 4) they gave a written informed consent.

Patients were excluded from the study if: 1) they were affected by drug abuse or drug dependence, delirium, mental retardation or organic mental disorders; 2) they had been previously treated with psychotropic medication; 3) they did not assure treatment adherence or they discontinued the treatment program for more than two consecutive visits (see follow-up evaluation).

\subsection{Assessment}

The period of enrolment lasted from January 1995 to December 1999. Patients were evaluated at two points in time, i.e., during the index hospital admission and then in 2010 .

\subsubsection{Baseline assessment}

The baseline evaluation was carried out within the first week of the index hospitalization. Socio-demographic variables recorded at baseline were: age, gender, years of education, marital and employment status, housing situation, age at onset of schizophrenia and the time passed from the onset of first psychotic symptoms or behavioural changes to the hospitalization where patients received treatment for the first time (see exclusion criteria).

To formulate the diagnosis, we used the information obtained from the Structured Clinical Interview for DSM-IV, carried out by trained psychiatrists, together with the information collected from family members, medical records and primary treating physicians.

The severity of symptoms was measured with the Positive and Negative Symptoms Scale (PANSS) [21]. According to the PANSS criteria, patients were defined as affected by positive schizophrenia (if they reported a score equal or higher than four in at least three items of positive scale and in less than three items of negative scale), negative schizophrenia (if they reported a score equal or higher than four in at least three items of negative scale and in less than three items of positive scale) or mixed schizophrenia (if they reported a score equal or higher than four in at least three items of positive and negative scales). 


\subsection{Follow-up evaluation}

After discharge from hospital, patients accessed to continuous and reasonably comprehensive public mental health services [22], where they attended nearly monthly visits.

During each visit their psychopathological condition, treatment adherence, medications prescription, use of illicit drugs were monitored from a clinical point of view. The number of psychotic episodes and time of hospitalization were also computed.

Patients were re-assessed in 2010 by a psychiatrist, blind to the baseline evaluation. The follow-up assessment consisted in the administration of SCID-IV and PANSS. After eight months patients were re-evaluated with the PANSS to verify whether the time criterion of 6 months requested for remission, as suggested by RSWG, was satisfied. Patients were defined in remission (R) or in nonremission (NR), according to the criteria (both severity and time criteria) proposed by the RSWG.

\subsection{Treatment}

All patients were treated with antipsychotic medications. The choice of medication was made on the base of clinical evaluation and according to the preference of patients and their relatives.

To maximize the treatment adherence, a medication control by relatives was recommended and the count of pills was performed at any time the medication was prescribed. In patients who did not assure a treatment adherence the use of a long-acting medication was proposed.

\subsection{Data analysis}

Before the start-up of the study and the follow-up evaluations an inter-rater reliability was calculated for the diagnosis of schizophrenia (baseline: $\mathrm{k}=.92$; follow-up: $\mathrm{k}=.95$ ) and for the PANSS total score (baseline: $\mathrm{k}=.82$; follow-up: $\mathrm{k}=.80$ ).

The clinical and socio-demographic features were compared in R and in NR using two-tailed Student's t test for continuous variables and $\chi^{2}$ test for categorical variables.

The change of symptom severity between baseline and follow-up assessments was evaluated in R and in NR using two-tailed Student's $t$ test for paired data.

A logistic regression analysis was next used to evaluate which factors may influence the likelihood to achieve remission. In this analysis, remission vs. non-remission

Table 1

Socio-demographic and clinical features of schizophrenic patients with or without remission.

\begin{tabular}{|c|c|c|c|c|c|c|c|c|}
\hline & \multicolumn{6}{|c|}{ Remission } & \multirow[t]{3}{*}{$x^{2}$} & \multirow[t]{3}{*}{$\mathrm{p}$} \\
\hline & \multicolumn{2}{|c|}{ Yes $\mathrm{n}=18$} & \multicolumn{2}{|c|}{ No $n=30$} & \multicolumn{2}{|c|}{ Total $n=48$} & & \\
\hline & $\mathrm{n}$ & $\%$ & $\mathrm{n}$ & $\%$ & $\mathrm{n}$ & $\%$ & & \\
\hline Male & 10 & 55.5 & 19 & 63.3 & 29 & 60.4 & & \\
\hline Marital status & & & & & & & 3.6 & .16 \\
\hline Never married & 13 & 72.2 & 22 & 73.3 & 35 & 72.9 & & \\
\hline Working status & & & & & & & 6.4 & .01 \\
\hline Never occupied & 4 & 22.2 & 18 & 60.0 & 22 & 45.8 & & \\
\hline Occupied & 14 & 77.8 & 12 & 40.0 & 26 & 54.2 & & \\
\hline Living status & & & & & & & 0.6 & .44 \\
\hline Living alone & 8 & 44.4 & 10 & 33.3 & 18 & 37.4 & & \\
\hline Living with someone & 10 & 55.6 & 20 & 66.7 & 30 & 62.5 & & \\
\hline Undifferentiated & 2 & 11.1 & 2 & 6.7 & 4 & 8.3 & & \\
\hline PANSS scores & & & & & & & 11.3 & .002 \\
\hline Positive & 15 & 83.3 & 10 & 33.3 & 25 & 52.1 & & \\
\hline Negative & 0 & 0.0 & 1 & 3.3 & 1 & 2.1 & & \\
\hline \multirow[t]{2}{*}{ Mixed } & 3 & 16.7 & 19 & 63.3 & 22 & 45.8 & & \\
\hline & \multicolumn{2}{|c|}{ Mean \pm SD } & \multicolumn{2}{|c|}{ Mean \pm SD } & \multicolumn{2}{|c|}{ Mean \pm SD } & $\mathrm{T}$ & $\mathrm{p}$ \\
\hline Age & \multicolumn{2}{|c|}{$41.0 \pm 11.3$} & \multicolumn{2}{|c|}{$46.5 \pm 14.1$} & \multicolumn{2}{|c|}{$43.2 \pm 11.1$} & 1.3 & .17 \\
\hline Age at onset & \multicolumn{2}{|c|}{$22.2 \pm 3.8$} & \multicolumn{2}{|c|}{$21.6 \pm 5.8$} & \multicolumn{2}{|c|}{$21.8 \pm 5.1$} & .3 & .70 \\
\hline DUP, weeks & \multicolumn{2}{|c|}{$18.0 \pm 4.5$} & \multicolumn{2}{|c|}{$19.7 \pm 4.4$} & \multicolumn{2}{|c|}{$19.0 \pm 4.5$} & 1.21 & .20 \\
\hline \multicolumn{9}{|l|}{ At follow-up } \\
\hline Duration years & \multicolumn{2}{|c|}{$16.3 \pm 5.1$} & \multicolumn{2}{|c|}{$16.0 \pm 4.8$} & \multicolumn{2}{|c|}{$16.2 \pm 4.9$} & .2 & .85 \\
\hline
\end{tabular}


(1 vs 0) was entered as the dependent variable, and the variables which showed a significant difference between $R$ and NR patients at baseline (see Table 1) were entered as indipendent variables.

\section{Results}

\subsection{Sample}

During the period of enrolment, 70 patients were consecutively admitted to the Psychiatric Clinic of the University of Parma for a first psychotic episode and discharged with a diagnosis of schizophrenia. Among them, 56 accepted to participate in the study.

In 2010 eight patients were lost to follow-up: six patients for the change of residence and two patients for refusing to further undergo evaluation. Therefore 48 patients were reassessed at follow-up.

The SCID-IV re-administration confirmed the stability of schizophrenia diagnosis in all 48 patients, and 18 of them satisfied remission criteria $(37.5 \%)(\mathrm{R})$, whereas the remaining 30 patients $(62,5 \%)$ (NR) did not reach remission. None of them was using illicit drugs.

Among remitted patients, 3 of them $(16.7 \%)$ were in complete symptomatic recovery, $9(50 \%)$ showed minimal symptom severity and $6(33.3 \%)$ had mild symptom severity at follow-up.

\subsection{Socio-demographic characteristics}

Only the working status was different in $\mathrm{R}$ and $\mathrm{NR}$ patients: $\mathrm{R}$ patients were more likely to be employed than NR patients $\left(\chi^{2}=6.4 ; p=0.01\right)$ (Table 1$)$. The duration of untreated psychosis was shorter in this sample (Table 1) and no difference was found between $\mathrm{R}$ and NR patients.

At follow-up, $\mathrm{R}$ and NR did not differ with respect to the duration of illness, whereas the number of psychotic episodes and time of hospitalization were higher in NR than in $\mathrm{R}$ (Table 1).

\subsection{Symptom severity}

At baseline a lesser symptom severity was found in $\mathrm{R}$ than in NR patients: all PANSS dimension scores (positive, negative and general psychopathology) were lower in R than in NR patients (Table 2). Eleven patients (22.9\%) did not have negative symptoms at the first episode.

At follow-up, symptoms severity had decreased in both groups: such decrease was statistically significant for PANSS positive and general psychopathology scores, but not for the negative symptoms score, in both $\mathrm{R}$ and NR patients (Table 2).

\subsection{Treatment}

Forty-one patients $(85.5 \%)$ were treated with oral antipsychotics and the remaining 7 patients $(14.5 \%)$ received a long-acting antipsychotic. A second-generation antipsychotic was administered in 34 patients $(70.8 \%)$ and a firstgeneration antipsychotic was used in 14 patients (29.2\%).

\subsection{Likelihood of remission}

The likelihood to achieve remission was predicted by the severity of general psychopathology and negative symptoms at onset and by the number of psychotic episodes during follow-up, while PANSS type of schizophrenia, PANSS positive symptoms and time of hospitalization did not exert any effect (Table 3).

\section{Discussion}

The current study evaluated the symptomatic course of schizophrenia by assessing 48 patients at their first episode and after a mean period of 16 years.

At follow-up, 18 patients $(37.5 \%)$ (R) satisfied the remission criteria (both severity and time criteria) proposed by the RSWG [11], whereas the remaining 30 patients did not achieve remission (NR).

Remitters and non-remitters showed significant psychopathological differences since their first episode of illness. $\mathrm{R}$ patients displayed milder positive and negative symptoms and a lower overall symptom severity at baseline than NR. These data are in keeping with previous findings of less severe early negative symptoms in R patients [19], but contradict the results of Ceskova et al. [20], who did not find any difference between $\mathrm{R}$ and NR at their first episode of

Table 2

Symptom severity (PANSS scores), in remitted (R) and in non-remitted (NR) patients.

\begin{tabular}{|c|c|c|c|c|c|c|c|c|}
\hline \multirow[t]{4}{*}{ PANSS symptoms } & \multicolumn{4}{|c|}{ Remission } & \multicolumn{4}{|c|}{ t-test for pared sample } \\
\hline & \multirow{2}{*}{\multicolumn{2}{|c|}{$\begin{array}{c}\text { Yes } \\
\mathrm{n}=18\end{array}$}} & \multirow{2}{*}{\multicolumn{2}{|c|}{$\begin{array}{c}\text { No } \\
\mathrm{n}=30\end{array}$}} & \multicolumn{2}{|c|}{ Yes vs no } & \multicolumn{2}{|c|}{ Baseline vs follow-up } \\
\hline & & & & & \multirow{2}{*}{$\frac{\text { Baseline }}{\mathrm{t}}$} & \multirow{2}{*}{$\frac{\text { Follow-up }}{\mathrm{t}}$} & \multirow{2}{*}{$\frac{\text { Yes }}{t}$} & \multirow{2}{*}{$\frac{\mathrm{No}}{\mathrm{t}}$} \\
\hline & Baseline & Follow-up & Baseline & Follow-up & & & & \\
\hline Positive & $24.8 \pm 3.7$ & $11.0 \pm 2.7$ & $28.1 \pm 4.0$ & $17.2 \pm 5.8$ & $2.7^{\#}$ & $4.2^{*}$ & $13.9^{*}$ & $11.5^{*}$ \\
\hline Negative & $17.8 \pm 3.1$ & $16.5 \pm 2.6$ & $25.3 \pm 4.4$ & $24.1 \pm 5.0$ & $6.1^{*}$ & $5.6^{*}$ & 1.6 & 1.7 \\
\hline General & $46.0 \pm 4.1$ & $32.5 \pm 5.5$ & $55.8 \pm 5.4$ & $41.7 \pm 6.9$ & $6.6^{*}$ & $4.7^{*}$ & $9.2 *$ & $12.9^{*}$ \\
\hline Total score & $88.8 \pm 6.2$ & $60.7 \pm 10.5$ & $109.2 \pm 10.0$ & $83.0 \pm 14.2$ & $7.7^{*}$ & $5.7^{*}$ & $10.5^{*}$ & $12.2^{*}$ \\
\hline
\end{tabular}


Table 3

Probability to reach remission at follow-up in patients with first episode of schizophrenia.

\begin{tabular}{|c|c|c|c|c|c|c|}
\hline \multicolumn{7}{|l|}{ Probability of remission } \\
\hline & $\mathrm{b}$ & $x^{2}$ wald & $\mathrm{p}$ & OR & CI $95 \%$ & $\mathrm{R}^{2}$ \\
\hline Step 1 & & & & & & .46 \\
\hline PANSS general psychopathology score & -.38 & 44.9 & $<.001$ & .68 & $.55-.84$ & \\
\hline Step 2 & & & & & & .56 \\
\hline PANSS negative score & -.49 & 5.6 & .01 & .60 & $.40-.91$ & \\
\hline PANSS general psychopathology score & -.28 & 5.5 & .01 & .75 & $.59-.95$ & \\
\hline Step 3 & & & & & & .68 \\
\hline No. of psychotic episodes follow-up & -1.3 & 7.1 & .008 & .68 & $.55-.84$ & \\
\hline PANSS negative score & -1.8 & 2.2 & .13 & .16 & $.01-1.78$ & \\
\hline PANSS general psychopathology score & -.49 & 2.0 & .14 & .60 & $.30-1.20$ & \\
\hline
\end{tabular}

In the logistic regression analysis remission vs. non remission ( 0 vs 1$)$ entered as dependent variable, whereas baseline PANSS positive, negative, and general psychopathological scores, PANSS types of schizophrenia, number of psychotic episodes and time of hospitalization during the follow-up period enterd as independent variables.

illness. However, while Ceskova et al. [20] included only men in their study, our sample had a $40 \%$ proportion of women, who are known to be characterized by a higher rate of remission [23]. Further, the achievement of remission was evaluated seven years after the first episode in the study by Ceskova et al. [20], and 16 years in the present study. The assessment of remission at different times of schizophrenia course may have contributed to such observed differences.

While in this sample positive and general psychopathology symptoms severity was found to improve 16 years after the first episode of illness, no change in negative symptoms severity occurred. These patterns of change (for positive and general symptoms) and stability (for negative symptoms) were observed in both R and NR, suggesting that R and NR patients share the same pattern of change across symptoms; thus, the clinical differences disclosed at their first episode were maintained during follow-up. Therefore, we can hypothesize that patients with milder symptoms severity at onset reached remission at follow-up when such symptoms improved, while a comparable degree of improvement did not translate into the achievement of remission in patients with more severe symptoms at onset of illness.

Concerning the effect of symptoms dimensions on remission, $\mathrm{R}$ patients exhibited more positive than negative symptoms at onset, since a "positive schizophrenia", defined according to the PANSS criteria, was more frequently diagnosed in $\mathrm{R}(83 \%)$ than in NR $(33 \%)(\mathrm{p}=0.002)$. However, logistic regression analysis demonstrated that the likelihood to develop remission is not influenced by the severity of positive symptoms at the first episode of illness, suggesting that positive symptoms at onset do not impact on later symptomatic remission. Of note, however, the number of psychotic episodes during follow-up reduced the likelihood of remission $(\mathrm{OR}=.68 ; \mathrm{CI} 95 \%=.55-.84)$ $(p=0.008)$, suggesting that the recurrence of positive symptoms during the course of illness may prevent remission.

Psychotic relapses occurring after schizophrenia onset may have a "toxic" effect on the brain because they are believed to induce a progressive brain loss. This structural brain change has been associated with the severity of psychotic symptoms and cognitive impairment as well as with the number and duration of psychotic episodes [24-26]. The results of this study may be interpreted as supporting those findings: a greater number of psychotic episodes could lead to a greater brain loss, which in turn increases the severity of psychotic symptoms thus reducing the likelihood of remission.

Therefore, the current study suggests that positive symptoms show a fluctuating course and their prognostic value varies as a function of their recurrence rather than of their initial severity, which in fact was found to decrease over time in this study sample, regardless of patients' remission status.

Conversely, negative symptoms were already present at onset in most patients (77\%), remained stable throughout the course of the illness, and exerted a negative effect on the achievement of remission. This finding is in keeping with recent studies indicating an association between higher negative symptoms at baseline and poor outcome [17-19]. Thus, in this sample of patients with schizophrenia, negative symptoms 1) had a relevant prognostic value, reducing the likelihood of remission; 2) were enduring, i.e., stable since the onset of schizophrenia until 16 years thereafter (looking refractory to the available antipsychotic treatments); 3) were primary since they neither worsened (for the addition of possible negative symptoms secondary to antipsychotic drugs), nor improved (for the reduction of negative symptoms secondary to the amelioration of positive symptoms) during a 16 year-old follow-up; 4) characterized each stage of the illness, being already apparent at onset in most patients and cannot be considered as a consequence of a chronic course. Taken together, these results support the classical view of negative symptoms as the cornerstone of schizophrenia [27].

In the present study, general psychopathology symptoms were negatively related to remission and their predicting value was even more relevant than that observed for negative 
symptoms. The PANSS "general psychopathology" dimension represents a heterogeneous dimension, including symptoms probably secondary to other dimensions (i.e. anxiety and agitation induced by positive symptoms), and symptoms that are a direct expression of the disease (i.e. avolition). This dimension could be more sensitive to illness severity, in a way that its predictive power is expression of the overall illness severity rather than of the effect of specific symptoms.

In summary, negative symptoms that are present at the onset of schizophrenia appear stable and associated with a poor long-term outcome in terms of symptomatic remission, while positive symptoms seem to have less prognostic value when assessed at onset, but their recurrence during the course of the disorder can impede long-term remission.

The present study has the following limitations.

First, symptom severity was assessed with the PANSS only at two points in time (i.e., at onset and after a mean period of 16 years of illness). However, it was clinically monitored at a monthly interval throughout the follow-up time. Thus, we can exclude that deep fluctuations of symptom severity had occured during the course of illness; rather, patients with mild symptoms at onset maintained the same symptom severity throughout the course of their illness. Nonethless, since schizophrenia is not a stable disorder and remission, as well as recovery, is for many patients a time limited occurrence, we cannot exclude that possible relapses may occur even after 16 years of follow-up in remitted patients.

Second, all patients enrolled in this study were hospitalized few months after the onset of illness, resulting in a short DUP (mean 19.0 weeks). This finding is probably due to the fact that a program of early detection and treatment of severe mental disorders, involving the collaboration between general practitioners and community mental health services, was active in the study catchment area. Therefore, the lack of effect of DUP on functional outcome in this sample can be explained by the early treatment intervention (i.e., within 30 weeks from the onset of psychotic symptoms or behavioural changes in all patients). In addition, the effect of the use of illicit drugs on the outcome could not be evaluated in the present study, since no participants had a diagnosis of substance use disorder during the followup period.

Third, even though the treatment was clinically monitored at each visit and strategies to prevent non-adherence were planned during the follow-up period, we cannot rule out that some patients were non-adherent to medications during the time between the visits, leading to incomplete control of symptoms.

Fourth, the current study includes patients who were strictly treatment adherent and substance use free. Thus, the results of the study are not applicable to overall schizophrenia patients.

Fifth, the study design did not provide a collection of prognostic indices at baseline through specific scales based upon clinical characteristics such as those developed by Vaillant [28] and Stephens [29].

In conclusion, this study suggests that symptomatic remission is a viable therapeutic outcome in schizophrenia, particularly in patients presenting with a less global severity and less severe negative symptoms at their first episode and who display few psychotic episodes over the course of their illness.

\section{References}

[1] Kraepelin E. Ein Lehrbuch fur Studierende und Aertze. Psychiatrie. 6. Auflage. Leipzig, Austria: Johann Ambrosins Barth; 1899.

[2] Lasser R, Nasrallah H, Helldin L, Peuskens J, Kane J, Docherty J, et al. Remission in schizophrenia: applying recent consensus criteria to refine the concept. Schizophr Res 2007;96:223-31.

[3] McGlashan TH. Premorbid adjustment, onset types, and prognostic scaling: still informative? Schizophrenia Bull 2008;34:801-5.

[4] Rund BR. Fully recovered schizophrenics: a retrospective study of some premorbid and treatment factors. Psychiatry 1990;53:127-39.

[5] Harrow M, Grossman L, Jobe TH, Herbener ES. Do patients with schizophrenia ever show periods of recovery? A 15-year multi-followup study. Schizophrenia Bull 2005;31:723-34.

[6] Van Os J, Burns T, Cavallaro R, Leucht S, Peuskens J, Helldin L, et al. Standardized remission criteria in schizophrenia. Acta Psychiat Scand 2006;113:91-5.

[7] Strauss JS, Carpenter Jr WT. Prediction of outcome in schizophrenia. I. Characteristics of outcome. Arch Gen Psychiat 1972;27:739-46.

[8] Leucht S, Davis JM, Engel RR, Kissling W, Kane JM. Definitions of response and remission in schizophrenia: recommendations for their use and their presentation. Acta Psychiat Scand Suppl 2009;119:7-14.

[9] Leucht S, Beitinger R, Kissling W. On the concept of remission in schizophrenia. Psychopharmacology 2007;194:453-61.

[10] Andreasen NC. Standardized remission criteria in schizophrenia. Acta Psychiatr Scand 2006;113:81.

[11] Andreasen NC, Carpenter WT, Kane JM, Lasser RA, Marder SR, Weinberger DR. Remission in schizophrenia: proposed criteria and rationale for consensus. Am J Psychiat 2005;162:441-9.

[12] Lambert M, De Marinis T, Pfeil J, Naber D, Schreiner A. Establishing remission and good clinical functioning in schizophrenia: predictors of best outcome with long-term risperidone long-acting injectable treatment. Eur Psychiat 2010;25:220-9.

[13] Liddle PF, Barnes TRE, Morris D, Haque S. Three syndromes in chronic schizophrenia. Brit J Psychiat 1989;155:119-22.

[14] Andreasen NC, Arndt S, Alliger R, Miller D, Flaum M. Symptoms of schizophrenia: methods, meanings, and mechanisms. Arch Gen Psychiat 1995;52:341-51.

[15] Ventura J, Hellemann GS, Thames AD, Joellner V, Nuechterlein KH. Symptoms as mediatiors of the reletionshio between neurocognition and functional outcome in schizophrenia. A meta-analysis. Schizophr Res 2009;113:189-99.

[16] Buoli M, Caldiroli A, Panza G, Altamura AC. Prominent clinical dimension, duration of illness and treatment response in schizophrenia: a naturalistic study. Psychiatry Invest 2012;9:354-60.

[17] Lang FU, Kösters M, Lang S, Becker T, Jäger M. Psychopathological long-term outcome of schizophrenia: a review. Acta Psychiat Scand 2013;127:173-82.

[18] Ayesa-Arriola R, Rodríguez-Sánchez JM, Pérez-Iglesias R, GonzálezBlanch C, PardoGarcía G, Tabares-Seisdedos R, et al. The relevance of cognitive, clinical and premorbid variables in predicting functional outcome for individuals with first-episode psychosis: A 3 year longitudinalstudy. Psychiat Res 2013, http://dx.doi.org/10.1016/j. psychres [Epub ahead of print]. 
[19] Kurihara T, Kato M, Reverger R, Tirta IG. Remission in schizophrenia: a community-based 6-year follow-up study in Bali. Psychiat Clin Neuros 2011;65:476-82.

[20] Ceskova E, Prikryl R, Kasparek T. Outcome in males with first-episode schizophrenia: 7-year follow-up. World J Biol Psychiatry 2011;12:66-72.

[21] Kay SR, Fiszbein A, Opler LA. The positive and negative syndrome scale (PANSS) for schizophrenia. Schizophrenia Bull 1987;13:261-76.

[22] Amaddeo F, Barbui C, Tansella M. State of psychiatry in Italy 35 years after psychiatric reform. Int Rev Psychiatr 2012;24:314-20.

[23] Carpiniello B, Pinna F, Tusconi M, Zaccheddu E, Fatteri F. Gender differences in remission and recovery of schizophrenic and schizoaffective patients: preliminary results of a prospective cohort study. Schizophr Res Treat 2012, http://dx.doi.org/10.1155/2012/576369 [Epub ahead of print 16 Jan 2012].

[24] Chakos M, Lieberman J, Hoffman E, Bradford D, Sheitman B. Effectiveness of second-generation antipsychotics in patients with treatment-resistant schizophrenia: a review and meta-analysis of randomized trials. Am J Psychiat 2001;158:518-26.

[25] Andreasen NC, Nopoulos P, Magnotta V, Pierson R, Ziebell S, Ho BC. Progressive brain change in schizophrenia: a prospective longitudinal study of first-episode schizophrenia. Biol Psychiat 2011;70:672-9.

[26] Andreasen NC, Liu D, Ziebell S, Vora A, Ho BC. Relapse duration, treatment intensity, and brain tissue loss in schizophrenia: a prospective longitudinal MRI study. Am J Psychiat 2013;170:609-15.

[27] Hafner H, Maurer K. Are there two types of schizophrenia? True onset and sequence of positive and negative syndromes prior to first admission. In: Marneros A, Andreasen NC, \& Tsuang MT, editors. Negative versus positive schizophrenia. Springer-Verlag: Springer-Verlag; 1991. p. 134-9.

[28] Vaillant GE. Prospective prediction of schizophrenic remission. Arch Gen Psychiatry 1964;11:509-18.

[29] Stephens JH, Astrup C, Mangrum JC. Prognostic factors in recovered and deteriorated schizophrenics. An J Psychiat 1966;122:1116-21. 